\title{
Electron structure of the equilibrium and metastable phases in superionic $\mathrm{Li}_{2} \mathrm{SiS}_{3}$
}

\author{
D.I. Bletskan, V.V. Vakulchak, K.E. Glukhov, O.A. Mykaylo \\ Uzhhorod National University, Physics Faculty, 46, Pidhirna str. 88000 Uzhhorod, Ukraine \\ Phone/fax: +38(031)-223-73-67; e-mail:crystal_lab457@yahoo.com
}

\begin{abstract}
The calculations of a band structure $E(k)$, the total $N(E)$ and partial densities of electron states, as well as spatial distribution of the valence charge $\rho(r)$ for the equilibrium $(e)$ and metastable $(m)$ phases of superionic $\mathrm{Li}_{2} \mathrm{SiS}_{3}$ were performed in a local approximation of the density functional theory (DFT) from the first principles for pseudopotentials in the basis of numerical pseudoatomic orbitals. The total density of states in the valence band calculated all over the Brillouin zone for both crystalline phases $\mathrm{Li}_{2} \mathrm{SiS}_{3}$ was compared to the experimental X-ray photoelectron spectra of the equilibrium, metastable and glassy phases. It is a good agreement between the calculation and experimental results for all bands of crystalline and glassy phases.
\end{abstract}

Keywords: superionics, lithium silicon sulfide, band structure, density of states, electron charge density map.

Manuscript received 07.11.12; revised version received 05.12.12; accepted for publication 26.01.13; published online 28.02.13.

\section{Introduction}

In $\mathrm{Li}_{2} \mathrm{~S}-\mathrm{SiS}_{2}$ system, two ternary compounds were found to exist: $\mathrm{Li}_{2} \mathrm{SiS}_{3}$, melting congruently at $T_{m}=1018 \pm$ $10 \mathrm{~K}$, and $\mathrm{Li}_{4} \mathrm{SiS}_{4}$, melting incongruently at $T_{m}=983 \pm$ $20 \mathrm{~K}[1,2] . \mathrm{Li}_{2} \mathrm{SiS}_{3}$ and $\mathrm{Li}_{4} \mathrm{SiS}_{4}$ ternary compounds are obtained by direct co-melting of $\mathrm{Li}_{2} \mathrm{~S}$ and $\mathrm{SiS}_{2}$ compounds in the ratio $1: 1$ and $2: 1$, respectively, in preevacuated sealed graphitized silica ampoules at temperatures not exceeding $1273 \mathrm{~K}[1,2]$. Depending on the melt cooling conditions, either an equilibrium phase, or the metastable crystalline one, or glassy $\mathrm{Li}_{2} \mathrm{SiS}_{3}$ can be obtained. The equilibrium crystalline phase is obtained upon rapid cooling to $993 \mathrm{~K}$ with subsequent annealing at the same temperature. The metastable phase is obtained under slow melt cooling from $T_{m}$ down to the room temperature for 5-6 hours. By melt quenching from $1053 \mathrm{~K}$ into water or liquid nitrogen, one can obtain glassy $\mathrm{Li}_{2} \mathrm{SiS}_{3}$. The glass-transition and crystallization temperatures of $\mathrm{Li}_{2} \mathrm{SiS}_{3}$ are $T_{g}=593 \mathrm{~K}$ and $T_{c}=723 \mathrm{~K}$, respectively [1].
In spite of the available data regarding structural studies of both crystalline phases $[1,3]$ and glassy $\mathrm{Li}_{2} \mathrm{SiS}_{3}$ by the direct techniques of X-ray [4] and neutron diffraction $[5,6]$ as well as vibrational spectroscopy $[7,8]$ and nuclear magnetic resonance $[9,10]$, physical properties of this compound have been studied quite poorly. Detailed investigations of the ionic conductivity were performed for both crystalline phases $[1,3]$ as well as stoichiometric [1] and non-stoichiometric glasses [9], also modified with $\mathrm{LiI}[11], \mathrm{LiBr}[12], \mathrm{Li}_{2} \mathrm{PO}_{4}[13,14]$. Analysis of the available experimental data on $\mathrm{Li}_{2} \mathrm{SiS}_{3}$ conductivity shows the electrical conductivity to increase by two orders of magnitude at the transition from the crystalline to glassy state, which is not typical for classical binary glass-forming compounds like $\mathrm{GeSe}_{2}, \mathrm{As}_{2} \mathrm{~S}_{3}, \mathrm{As}_{2} \mathrm{Se}_{3}$. For these compounds, the crystalglass transition is accompanied by a decrease in electrical conductivity by one or two orders [15]. From the practical viewpoint, the existence of the high ionic conductivity $\left(1 \cdot 10^{-4} \Omega^{-1} \mathrm{~cm}^{-1}\right)$ in glassy $\mathrm{Li}_{2} \mathrm{SiS}_{3}[1]$ is very important, and its value can be increased by an 
incorporation of $\mathrm{LiI}[11], \mathrm{LiBr}[12]$, or $\mathrm{Li}_{2} \mathrm{PO}_{4}[13,14]$ modifiers. It is the high ionic conductivity that makes lithium-containing silicon sulfide glasses (glassy silicon sulfides) promising solid electrolytes for lithium-ion batteries [11] and causes an increased interest towards these glasses.

With regard to the optical properties and theoretical calculations of the electron structure of the equilibrium and metastable crystalline phases $\mathrm{Li}_{2} \mathrm{SiS}_{3}$, such studies have not been carried out before. In this work, we report on the density functional theory (DFT) calculations of electron energy structure, the total $N(E)$ and partial densities of electron states for the equilibrium and metastable phases $\mathrm{Li}_{2} \mathrm{SiS}_{3}$, as well as the spatial distribution of valence charges in the unit cells of these modifications. The calculations of the total density of states $N(E)$ were compared with the available data of Xray photoelectron spectra (XPS) for the crystals of both phases and the glassy $\mathrm{Li}_{2} \mathrm{SiS}_{3}$.

\section{Crystal structure of the equilibrium and metastable phases of $\mathrm{Li}_{2} \mathrm{SiS}_{3}$}

The equilibrium phase $\mathrm{Li}_{2} \mathrm{SiS}_{3}(e)$ is isostructural to $\mathrm{Li}_{2} \mathrm{SiO}_{3}[16]$ and crystallizes in an orthorhombic lattice with the parameters $a=11.664 \AA, b=6.735 \AA$, and $c=$ $5.926 \AA, \mathrm{Cmc}_{1}$ space group $[1,3]$. The crystal structure of the equilibrium phase and its projection on the $\mathrm{YZ}$ plane are shown in Figs 1a and 1b, respectively. The unit cell contains four formula units (Fig. 2). Its volume is $466 \AA^{3}$, and the theoretically calculated density is $1.97 \mathrm{~g} / \mathrm{cm}^{3}$. Experimental measurements of the density for a slowly cooled sample gave the value of $1.87 \mathrm{~g} / \mathrm{cm}^{3}$ [5]. The main structural elements of $\mathrm{Li}_{2} \mathrm{SiS}_{3}$ crystal structure are $\left[\mathrm{SiS}_{4}\right]$ tetrahedra linked by common vertices in infinite zigzag chains extending along the $c$ axis (Fig. 1b). Each subsequent $\left[\mathrm{SiS}_{4}\right]$ tetrahedron in the chain is turned by $180^{\circ}$ with respect to the previous one. Parallel infinite chains are linked together by weak Van der Waals forces, $\mathrm{Li}^{+}$ions being located in the Van der Waals space. The $\mathrm{Si}-\mathrm{S}-\mathrm{Si}$ valence bond angles are between $138^{\circ}$ and $143^{\circ}$. Eight lithium ions are located in interstitial sites surrounded by five sulfur ions. As noted in Ref. [1], there are two types of sixfold interstitial sites in $\mathrm{Li}_{2} \mathrm{SiS}_{3}$ structure, differing in the shape of octahedra and their occupation by lithium ions. An important element of $\mathrm{Li}_{2} \mathrm{SiS}_{3}$ crystal structure is the presence of octahedral interstitial sites forming conductive "tunnels" along the $c$ axis that provide the conducting channels along which lithium ions move under an external electric field. This factor determines a rather high ionic conductivity value in this phase.

The metastable crystalline phase $\mathrm{Li}_{2} \mathrm{SiS}_{3}(m)$ also possesses orthorhombic structure typical for the equilibrium one, but with different lattice parameters: $a=11.436 \AA, b=6.605 \AA$, and $c=6.487 \AA$ [1]. The comparison of crystal lattice parameters for the equilibrium and metastable phases shows that the $a$ and $b$ parameters of the metastable phase are smaller than the same parameters of the equilibrium one. On the other hand, the $c$ parameter is greater in the metastable phase than in the equilibrium one. Consequently, the chains of vertex-linked $\left[\mathrm{SiS}_{4}\right]$ tetrahedra in the metastable phase are more elongated along the $c$ axis than in the equilibrium phase, so that $a$ and $b$ lattice parameters decrease, but the $c$ parameter and the valence bond angle $\mathrm{Si}-\mathrm{S}-\mathrm{Si}$ increase. Besides, the unit cell volume increases by $5 \%$.

\section{Calculation method}

The calculations of the energy band structure were carried out within the framework of the density functional theory $[17,18]$ in the local approximation for the account of exchange and correlation effects $[19,20]$, using SIESTA software [21,22]. Linear combinations of atomic orbitals were used as a fundamental calculation basis. The periodic structure of crystal is taken into

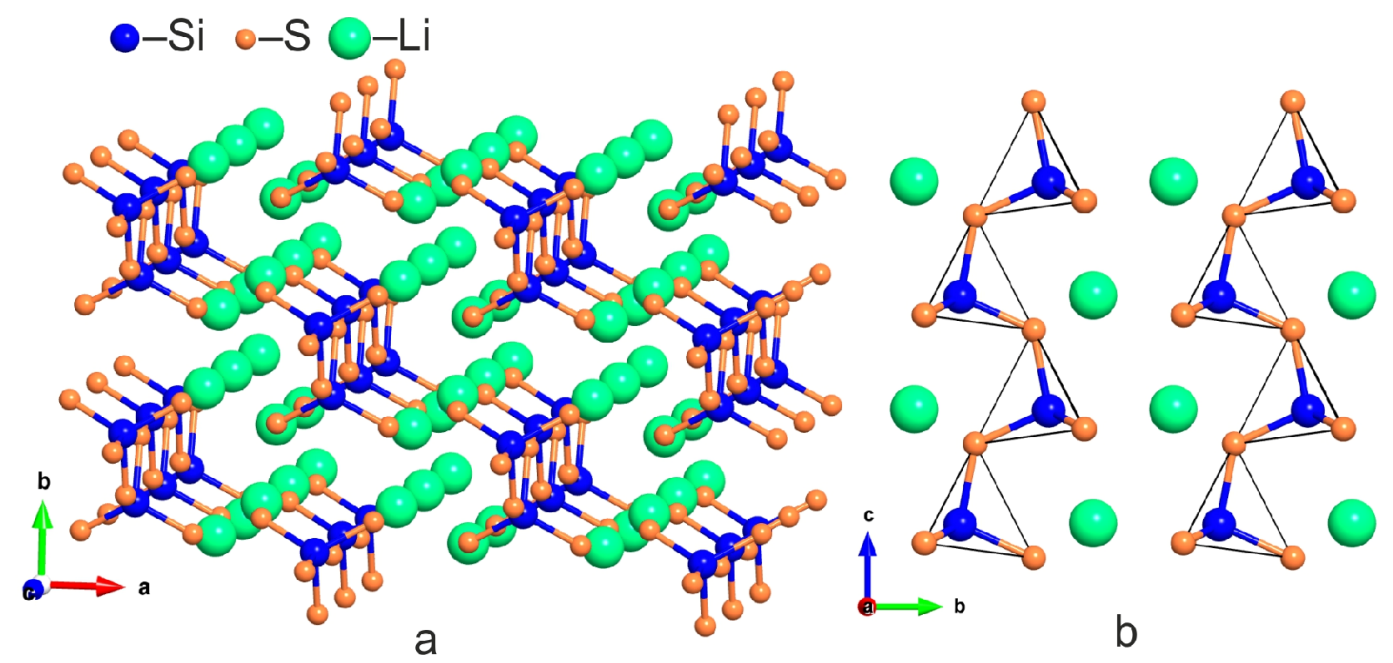

Fig. 1. The crystal structure $(a)$ and its projection on the $\mathrm{XZ}$ plane $(b)$ for the equilibrium phase of $\mathrm{Li}_{2} \mathrm{SiS}_{3}$. 
Table. Parameters of the band spectrum for the equilibrium and metastable phases in $\mathrm{Li}_{2} \mathrm{SiS}_{3}$.

\begin{tabular}{|c|c|c|c|c|c|c|c|c|c|c|c|c|c|}
\hline Compound & $\begin{array}{c}E_{V B}, \\
\mathrm{eV}\end{array}$ & $k_{V}$ & $k_{C}$ & $\begin{array}{c}E_{g i}, \\
\mathrm{eV}\end{array}$ & $\begin{array}{c}E_{g d}, \\
\mathrm{eV}\end{array}$ & $\begin{array}{c}E_{V B 1}, \\
\mathrm{eV}\end{array}$ & $\begin{array}{c}E_{V B 2}, \\
\mathrm{eV}\end{array}$ & $\begin{array}{c}E_{V B 3}, \\
\mathrm{eV}\end{array}$ & $\begin{array}{c}E_{V B 4}, \\
\mathrm{eV}\end{array}$ & $\begin{array}{c}\Delta E_{1}, \\
\mathrm{eV}\end{array}$ & $\begin{array}{c}\Delta E_{2}, \\
\mathrm{eV}\end{array}$ & $\begin{array}{c}\Delta E_{3}, \\
\mathrm{eV}\end{array}$ & $\begin{array}{c}E_{C B 1}, \\
\mathrm{eV}\end{array}$ \\
\hline $\mathrm{Li}_{2} \mathrm{SiS}_{3}(\mathrm{e})$ & 13.83 & $\mathrm{Z}$ & $\mathrm{G} \rightarrow \mathrm{F}$ & 2.82 & 2.99 & 2.41 & 2.26 & 2.02 & 2.69 & 0.19 & 0.55 & 3.79 & 0.98 \\
\hline $\mathrm{Li}_{2} \mathrm{SiS}_{3}(\mathrm{~m})$ & 13.4 & $\mathrm{~F}$ & $\mathrm{G} \rightarrow \mathrm{F}$ & 2.52 & 2.67 & 2.08 & 2.19 & 1.92 & 2.34 & 0.26 & 0.75 & 3.86 & 1.29 \\
\hline
\end{tabular}

account through the boundary conditions at the unit cell boundaries. First-principle atomic norm-preserving pseudopotentials $[23,24]$ for electron configurations of $\mathrm{Si}\left([\mathrm{Ne}] 3 s^{2} 3 p^{2}\right)$ and $\mathrm{S}\left([\mathrm{Ne}] 3 s^{2} 3 p^{4}\right)$ atoms were used in the calculations. These states belong to the valence shells while $[\mathrm{Ne}]$ is related to the core.

The plane wave effective cutoff energy $E_{c u t}=$ $200 \mathrm{Ry}$ for the self-consistent calculation (SCF) was chosen in such a way to obtain cell energy convergence not worse than $0.001 \mathrm{Ry} /$ atom. Then the basis contained about 30500 atomic orbitals. The density of $k$-points mesh in the reciprocal space for the self-consistent calculation was chosen from the same reason. The electron density was calculated by interpolation between the nodes of $5 \times 5 \times 6$ grid in the reciprocal space. The total and partial densities of electron states were determined by the modified tetrahedra method, for which the energy spectrum and wave functions were calculated on a $k$-grid containing 75 points. Integration over the irreducible part of the Brillouin zone was performed using the method of special $k$-points $[25,26]$.

\section{Results and discussion}

\subsection{Band structure and the nature of electron states}

The band structure calculation was performed for the high symmetry points and directions in the Brillouin zone shown in Fig. 3.

The band structure of the equilibrium and metastable crystalline phases of $\mathrm{Li}_{2} \mathrm{SiS}_{3}$ calculated without spin-orbit interaction is presented in Fig. 4. The base energy corresponds to the highest occupied state. The energy band structures of the two phases are qualitatively similar to each other and have characteristic topology with four clearly separated energy regions possessing a "doublet" structure. The valence bands of both crystals possess weak dispersion and form separate energy subbands (VBI, VBII, VBIII, VBIV) that correspond to distinct maxima in $N(E)$ (Fig. 5). The main quantitative parameters of the electron spectra for both crystal structures of $\mathrm{Li}_{2} \mathrm{SiS}_{3}$ are presented in Table. We used the following notations in the table: $E_{V B}$ is the total valence band width, $k_{V}$ is the point of the Brillouin zone corresponding to the valence band maximum, $k_{C}$ is the bottom of the lower unoccupied band, $E_{V B 1}, E_{V B 2}$, $E_{V B 3}$, and $E_{V B 4}$ are the widths of allowed energy bands for the first, second, third, and fourth subbands of the valence band, numbering the bands started from the top, $\Delta E_{1}, \Delta E_{2}$, and $\Delta E_{3}$ are the energy gap bandwidth, $E_{C B 1}$ is the width of the lower unoccupied subband, $E_{g i}$ is the indirect band gap, $E_{g d}$ is the direct band gap.

The top of the valence band (the absolute maximum) of the equilibrium $\mathrm{Li}_{2} \mathrm{SiS}_{3}$ is in the $\mathrm{Z}$ point, and for the metastable phase it is in the vertex point $F$ of the Brillouin zone. The bottom of the conduction band (absolute minimum) in both phases is not in a highsymmetry point, but at the Brillouin zone edge in the direction G-F at the distance of ca. 1/3 from the $G$ point. The band extrema have the following coordinates in the basic reciprocal lattice vector units: $Z(0,0,1 / 2), F(1 / 3$, $1 / 3,0), \mathrm{G}(1 / 3,1 / 3,1 / 2)$. Consequently, both $\mathrm{Li}_{2} \mathrm{SiS}_{3}$ crystals are indirect-gap superionic semiconductors with calculated indirect band gap $E_{g i}=2.82 \mathrm{eV}$ for the equilibrium phase of $\mathrm{Li}_{2} \mathrm{SiS}_{3}$ and $E_{g i}=2.52 \mathrm{eV}$ for the metastable one. It should be noted that the calculations in the local density approximation within the framework of the density functional theory always give underestimated band gap values.

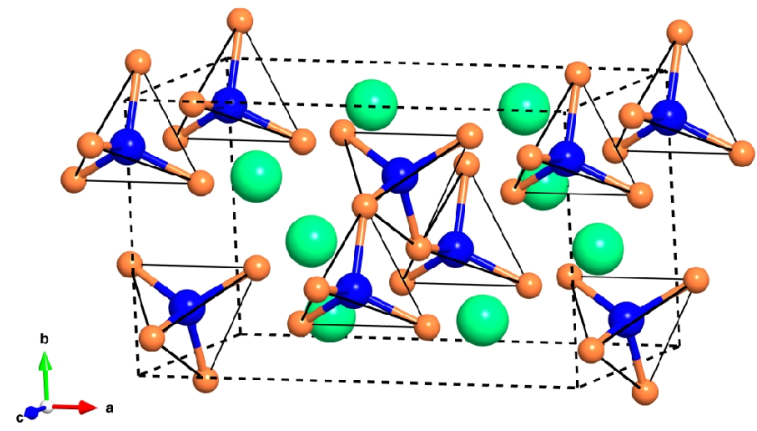

Fig. 2. The unit cell of the $\mathrm{Li}_{2} \mathrm{SiS}_{3}$ equilibrium phase.

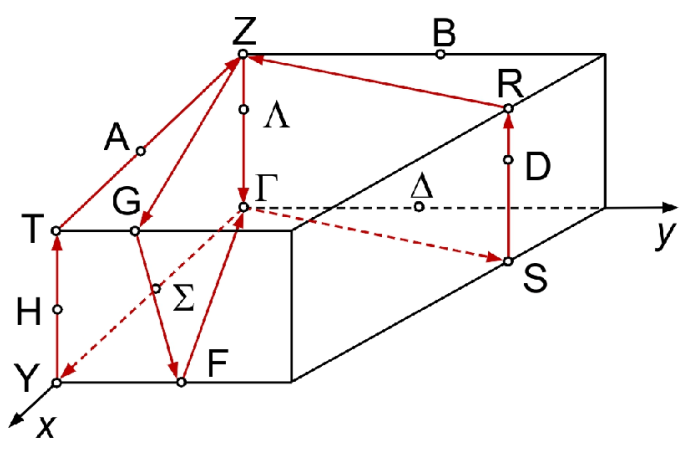

Fig. 3. The Brillouin zone of orthorhombic $\mathrm{Li}_{2} \mathrm{SiS}_{3}\left(\mathrm{Cmc}_{1}\right)$.

(C) 2013, V. Lashkaryov Institute of Semiconductor Physics, National Academy of Sciences of Ukraine 

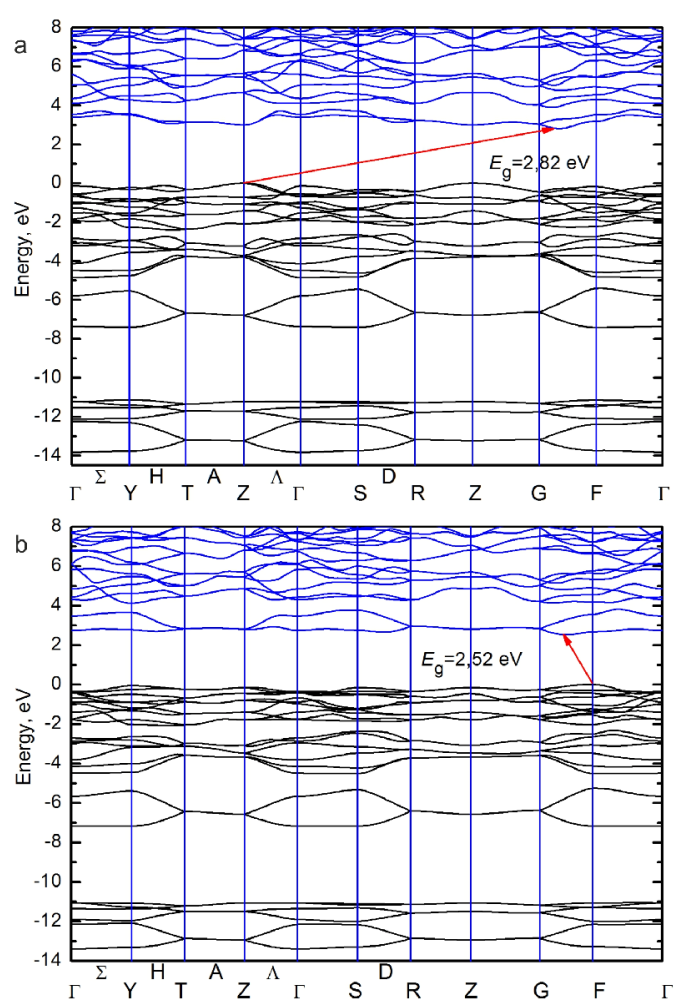

Fig. 4. The band structure of the equilibrium orthorhombic $(a)$ and metastable $(b)$ phases in $\mathrm{Li}_{2} \mathrm{SiS}_{3}\left(\mathrm{Cmc}_{1}\right)$.

Calculations of the total $N(E)$ and partial densities of electron states provide information on the contribution of atomic orbitals into the crystalline states, which for both modifications of $\mathrm{Li}_{2} \mathrm{SiS}_{3}$ are qualitatively similar (Fig. 5). The main contribution to the valence band in both crystalline phases is made by $s$ - and $p$-states of sulfur. The analysis of the total and partial densities of electron states for both crystals shows that the lowest valence subband VBIV is formed predominantly by $3 s-$ states of sulfur with a small admixture of $3 s$ - and $3 p$ states of silicon and $2 p$-states of lithium. The VBIII subband is formed by roughly equal similar contribution of hybridized Si $3 s$ - and S $3 p$-states.

$3 p$-orbitals of sulfur mixed with $3 p$-orbitals of silicon and $2 s$ - and $2 p$-states of lithium are mostly involved in the formation of the VBII subband. Finally, $3 p$-orbitals of sulfur with a small admixture of $3 d$-states of silicon and $2 s$ - and $2 p$-states of lithium make the main contribution to the topmost valence VBI subband.

The lowest unoccupied CBI subband is separated from other unoccupied bands by a gap. The lower conduction band is characterized by hybridization of all silicon states with $3 p$ - and $3 d$-states of sulfur as well as $2 s$ - and $2 p$-states of lithium. The resulting position of individual energy subbands of silicon, sulfur and lithium in $\mathrm{Li}_{2} \mathrm{SiS}_{3}$ can be explained by the preserved atomic genesis of these subbands with account of effective charges on atoms in the crystal. A higher electronegativity of $\mathrm{S}$ atoms compared to the $\mathrm{Si}$ ones leads to the smaller binding energies for the $s$ - and $p$ states of sulfur, thus determining the relative positions of $\mathrm{S}$ and $\mathrm{Si}$ subbands in the valence band of $\mathrm{Li}_{2} \mathrm{SiS}_{3}$. From this assumption on the reasons for the relative positions of the $\mathrm{Si}$ and $\mathrm{S}$ subbands in the valence band $\mathrm{Li}_{2} \mathrm{SiS}_{3}$, it follows directly that the fraction of ionicity in the $\mathrm{Si}-\mathrm{S}$ chemical bond is quite substantial.

X-ray photoelectron spectroscopy (XPS) is an informative experimental technique for studying the electron structure of crystalline and glassy superionics. For both crystalline phases and glassy $\mathrm{Li}_{2} \mathrm{SiS}_{3}$ experimental X-ray photoelectron spectra are available, which provide information on the energy distribution of electron states in the valence band. Comparison of the theoretically calculated total and partial densities of
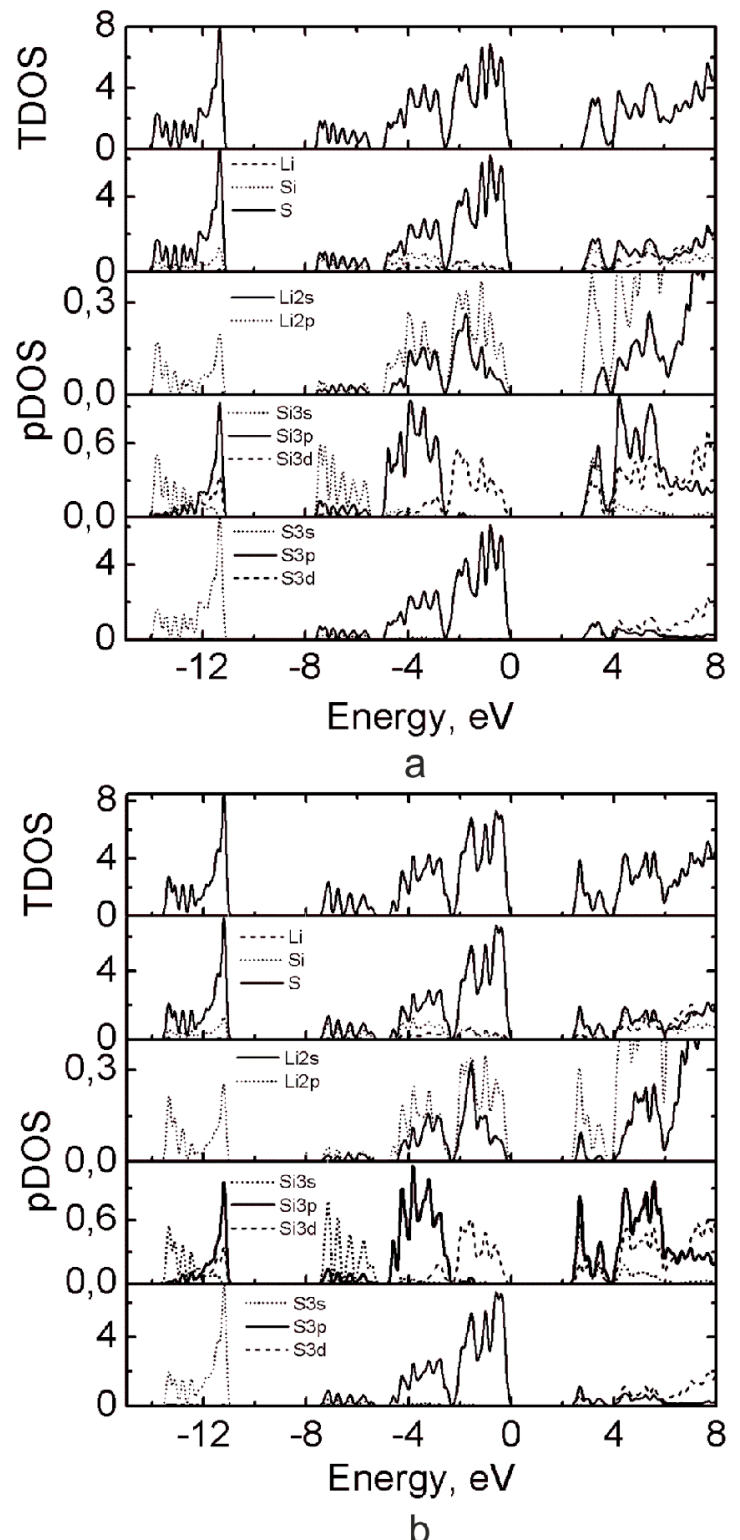

Fig. 5. Total and partial densities of electron states for the equilibrium $(a)$ and metastable $(b)$ phases in $\mathrm{Li}_{2} \mathrm{SiS}_{3}$ $\left(\mathrm{Cmc} 2_{1}\right)$. 


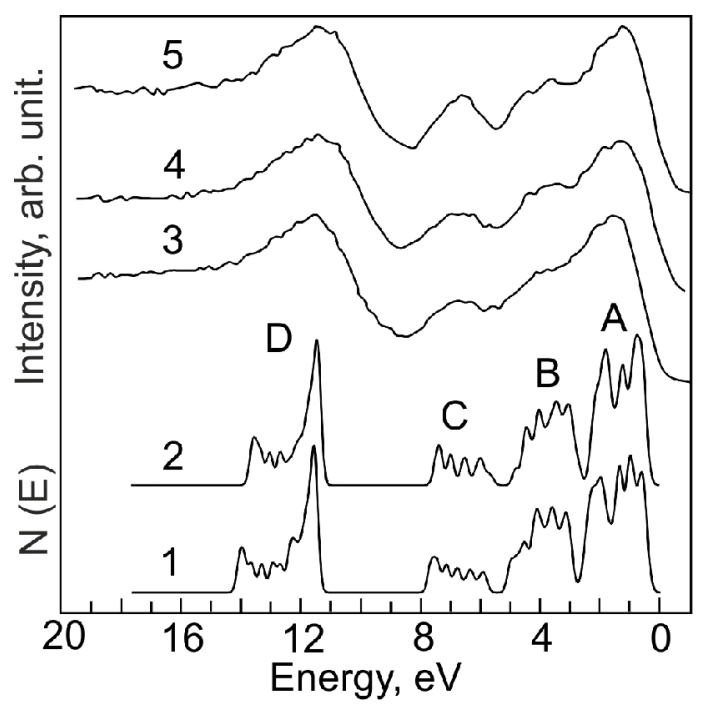

Fig. 6. The calculated total density of electron states $N(E)$, XPS spectra (3-5) [27] of the equilibrium $(1,5)$, metastable $(2,4)$, and glassy $(3)$ phases in $\mathrm{Li}_{2} \mathrm{SiS}_{3}$.

electron states with the experimental XPS spectra enables the features of the X-ray photoelectron spectra to be genetically linked with the states of certain symmetry inherent to $\mathrm{Si}$ and $\mathrm{S}$ atoms, as well as the influence of local environment on the studied valence band structure.

Fig. 6 presents comparison of the experimental Xray photoelectron spectra (excitation by monochromatic $\mathrm{Al} K_{\alpha}$ radiation with the energy $h v=1486.6 \mathrm{eV}$ ) for both crystalline phases and glassy $\mathrm{Li}_{2} \mathrm{SiS}_{3}$ [27] with the calculated total densities of states $N(E)$ in the valence bands of the equilibrium and metastable crystalline phases. It is clearly seen from Fig. 6 that the relative intensities of the main peaks and their energy positions in the XPS spectra of both crystalline phases as well as the glassy phase are in a good agreement with the theoretical curves $N(E)$ of the crystals. Consequently, the observed good agreement of the experimental X-ray photoelectron spectra of the crystals and glass with the corresponding calculated $N(E)$ spectra enables us to conclude that the DFT band calculations give a qualitatively correct picture of $\mathrm{Li}_{2} \mathrm{SiS}_{3}$ electron structure. Additionally, the similarity of XPS spectra for both $\mathrm{Li}_{2} \mathrm{SiS}_{3}$ crystals and glass means that the main features of the electron structures of all three phases are determined by the short-range order in the atom arrangement. The analysis of the fine structure of X-ray photoelectron spectra and theoretically calculated local densities of $\mathrm{Si} s-, p$-, and $\mathrm{S} p$-states undoubtedly testifies for a significant mixing of these states and demonstrates the presence of a covalent component in the chemical bond between $\mathrm{Si}$ and $\mathrm{S}$ atoms in the $\left[\mathrm{SiS}_{4}\right]$ coordination tetrahedra that form the infinite chains.

\subsection{Spatial distribution of the electron charge}

The distribution of electrons in $\mathrm{Li}_{2} \mathrm{SiS}_{3}$ crystalline phases can be described, at least in the first approximation, by electron charge density mapping. Whereas $\rho(r)$ is a function in the three-dimensional space, the results can be conveniently shown on a plane in the form of electron charge density maps [28]. However, the tetrahedral structure of the short-range order of the crystalline phases in $\mathrm{Li}_{2} \mathrm{SiS}_{3}$ encumbers 2D mapping. In this case, it is most convenient to present the electron configurations in a separate $\left[\mathrm{SiS}_{4}\right]$ tetrahedron in the plane containing two sulfur atoms and one silicon atom. Since $\left[\mathrm{SiS}_{4}\right]$ tetrahedra linked in the chain possess two bridging and two non-bridging sulfur atoms, it is reasonable to build two planes: one across the silicon atom and two bridging sulfur atoms and the second one - across the silicon atom and two non-bridging sulfur atoms. The distribution of the valence charge between the silicon ions and the bridging and non-bridging sulfur ions belonging to the same $\left[\mathrm{SiS}_{4}\right]$ tetrahedron is illustrated by the electron density maps in Figs $7 \mathrm{a}$ and $7 \mathrm{~b}$, respectively. The contours of the contribution of the core electrons have a very high concentration near the $\mathrm{Si}$ and $\mathrm{S}$ atom locations. Comparison of the maps (Figs 7a
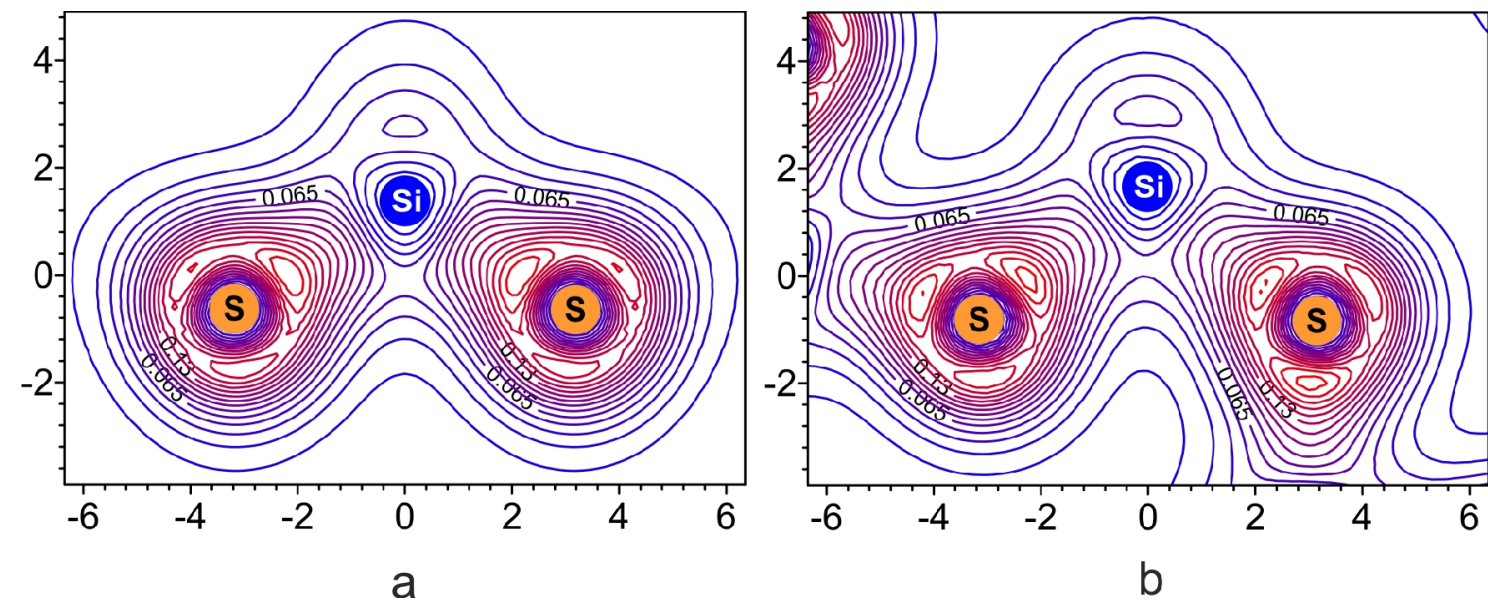

Fig. 7. Spatial distribution of the charge density in the valence band of $\mathrm{Li}_{2} \mathrm{SiS}_{3}$ in the plane of $\mathrm{S}-\mathrm{Si}-\mathrm{S}$ bond containing: nonbridging $(a)$ and bridging $(b)$ sulfur atoms. 


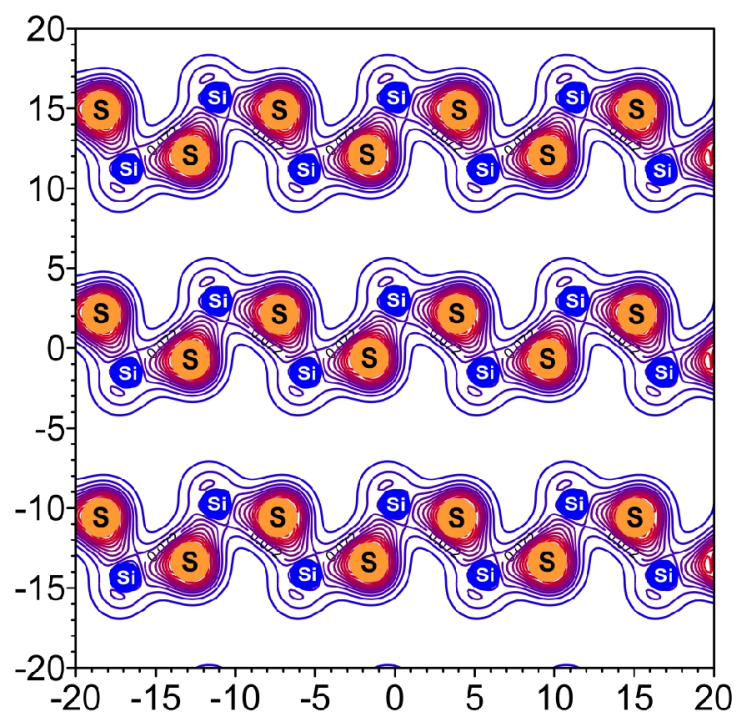

a

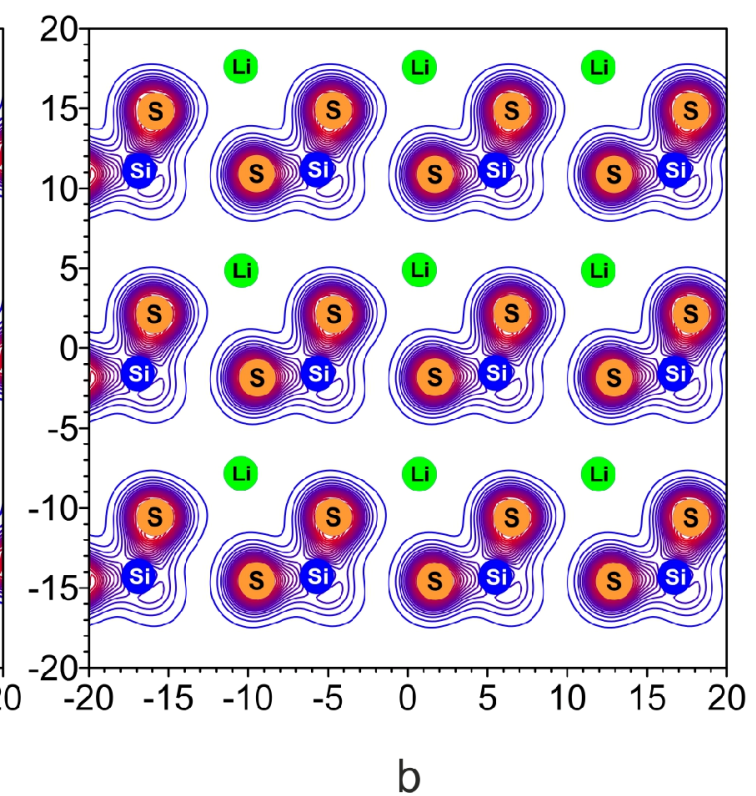

b

Fig. 8. Maps of the electron density: $(a)$ in the plane $(1 \overline{1} 0)$ built across the silicon atoms and bridging sulfur atoms in a [SiS 4 tetrahedron; $(b)$ in the plane passing through the silicon, non-bridging sulfur, and lithium atoms in the $\mathrm{Li}_{2} \mathrm{SiS}_{3}$ equilibrium phase.

and $7 \mathrm{~b}$ ) shows both planes have common contours of the density $\rho(r)$ encompassing sulfur atoms and anions in general as well as sulfur and silicon atoms. The difference consists in the fact that the plane passing through the non-bridging sulfur atoms contains only common contours encompassing sulfur atoms and the same silicon atom. These contours extend from each of the sulfur atom only along one $\mathrm{Si}-\mathrm{S}$ bond line (Fig. 7a). The plane passing through two non-bridging sulfur atoms (Fig. 7b) contains common contours encompassing the same bridging sulfur atom of one tetrahedron and two silicon atoms belonging to two neighboring tetrahedra.

As a result of interaction between sulfur and silicon atoms, the electron charge is redistributed from the silicon to sulfur atoms along the $\mathrm{Si}-\mathrm{S}$ bond line, thus providing the covalent component of the chemical binding within $\left[\mathrm{SiS}_{4}\right]$ tetrahedra belonging to the same chain. The transfer of a significant part of its valence charge from silicon atoms to sulfur favors hybridization of $\mathrm{Si} 3 p$-orbitals with $\mathrm{S} 3 s$-orbitals. Ionic binding is characterized by the increase in charge around the anion and the charge decrease at the covalent bond between ions inside the tetrahedron. In both crystalline phases, anisotropy of the charge density is observed, reflecting the difference in the interaction between $\mathrm{S}$ ions belonging to the same chain with the sulfur atoms of neighboring chains. Ions from two different neighboring chains interact extremely weakly (Fig. 8a).

The weak participation of lithium atoms in formation of electron states can be seen from the contour maps in the plane passing through the silicon atoms, non-bridging sulfur, and lithium atoms (Fig. 8b). Their shape clearly shows that contributions of sulfur atoms occupy much wider space than those from silicon and by far wider than those from lithium. The small charge density around $\mathrm{Li}^{+}$ions and the presence of channels between the chains with very low charge density along the $c$ axis provide the high mobility of lithium ions along these channels and, as a consequence, the high ionic conductivity of both crystalline phases $\mathrm{Li}_{2} \mathrm{SiS}_{3}$.

\section{Conclusions}

The band structure of two polymorphic forms of crystalline $\mathrm{Li}_{2} \mathrm{SiS}_{3}$ superionic conductor was calculated by non-empirical density functional method in the local approximation. This made it possible to elucidate the main features of the electron structure of this compound. Both crystals are indirect-gap superionic semiconductors with the calculated band gap values of $2.82 \mathrm{eV}$ for the equilibrium phase and $2.52 \mathrm{eV}$ for the metastable phase.

The profiles of the total densities of electron states $N(E)$ for both crystalline phases are similar and correlate with all the features in the experimental XPS spectra of the corresponding crystals. Meanwhile, X-ray photoelectron spectra of the glass and the metastable crystalline phase are quite similar. Therefore, the electron structure of $\mathrm{Li}_{2} \mathrm{SiS}_{3}$ superionic is determined by the short-range order.

Distribution of the electron density over the unit cell is anisotropic. The electron density maps built for a number of crystallographic planes demonstrate that the ionic conductivity is indeed carried out along the "tunnels" between the infinite chains characterized by the minimum electron density.

\section{(C) 2013, V. Lashkaryov Institute of Semiconductor Physics, National Academy of Sciences of Ukraine}




\section{References}

1. B.T. Ahn and R.A. Huggins, Preparation, structures and conductivities of $\mathrm{Li}_{2} \mathrm{SiS}_{3}$ phases // Mat. Res. Bull. 25, p. 381-389 (1990).

2. B.T. Ahn and R.A. Huggins, Phase behavior and conductivity of $\mathrm{Li}_{2} \mathrm{SiS}_{3}$ composition // Solid State Ionics, 46, p. 237-242 (1991).

3. B.T. Ahn and R.A. Huggins, Synthesis and lithium conductivities of $\mathrm{Li}_{2} \mathrm{SiS}_{3}$ and $\mathrm{Li}_{4} \mathrm{SiS}_{4} / /$ Mat. Res. Bull. 24, p. 889-897 (1989).

4. K. Muruganandam and M. Seshasayee, Structural investigation of $\mathrm{Li}_{2} \mathrm{SiS}_{3}$ glass using X-ray RDF // Solid State Communs. 95, p. 499-502 (1995).

5. D.L. Price and A.J.G. Ellison, Atomic structure and dynamics of fast-ion conducting glasses // J. NonCryst. Solids, 177, p. 293-298 (1994).

6. S.R. Elliott, Isotopic-substitution neutron diffraction as a probe of the structural environment of cations in superionic glasses // Solid State Ionics, 105, p. 39-45 (1998).

7. A. Pradel and M. Ribes, Lithium chalcogenide conductive glasses // Mat. Chem. Phys. 23, p. 121142 (1989).

8. J.H. Kennedy and Y. Yang, Glass-forming region and structure in $\mathrm{SiS}_{2}-\mathrm{Li}_{2} \mathrm{~S}-\mathrm{Lix}(x=\mathrm{Br}, \mathrm{I}) / /$ J. Solid State Chem. 69, p. 252-257 (1987).

9. H. Eckert, Z. Zhang and J.H. Kennedy, Glass formation in non-oxide chalcogenide systems. Structural elucidation of $\mathrm{Li}_{2} \mathrm{~S}-\mathrm{SiS}_{2}-\mathrm{LiI}$ solid electrolytes by quantitative ${ }^{29} \mathrm{Si},{ }^{6} \mathrm{Li}$ and ${ }^{7} \mathrm{Li}$ high resolution solid state NMR methods // J. NonCryst. Solids, 107, p. 271-282 (1989).

10. A. Pradel, G. Taillades, M. Ribes and H. Eckert, Eckert H. ${ }^{29} \mathrm{Si}$ NMR structural studies of ionically conductive silicon chalcogenide glasses and model compounds // J. Non-Cryst. Solids, 188, p. 75-86 (1995).

11. A. Pradel and M. Ribes, Electrical properties of lithium conductive silicon sulfide glasses prepared by twin roller quenching // Solid State Ionics, 1819, p. 351-355 (1986).

12. S. Sahami, S.W. Shea and J.H. Kennedy, Preparation and conductivity measurements of $\mathrm{SiS}_{2}-\mathrm{Li}_{2} \mathrm{~S}-\mathrm{LiBr}$ lithium ion conductive glasses // $J$. Electrochem. Soc. 132, p. 985--986 (1985).

13. S. Kondo, K. Takada and Y. Yamamura, New lithium ion conductors based on $\mathrm{Li}_{2} \mathrm{~S}_{-} \mathrm{SiS}_{2}$ system // Solid State Ionics, 53-56, p. 1183-1186 (1992).
14. N. Aotani, K. Iwamoto, K. Takada and S. Kondo, Synthesis and electrochemical properties of lithium ion conductive glass, $\mathrm{Li}_{3} \mathrm{PO}_{4}-\mathrm{Li}_{2} \mathrm{~S}-\mathrm{SiS}_{2} / /$ Solid State Ionics, 68, p. 35-39 (1994).

15. D.I. Bletskan, N.V. Polazhinets and D.V. Chepur, Photoelectric properties of crystalline and glassy $\mathrm{GeSe}_{2}$ // Fizika i tekhnika poluprov. 18, p. 223-228 (1984), in Russian.

16. K.-F. Hesse, Refinement of the crystal structure of lithium polysilicate // Acta Crystallogr. B, 33, p. 901-902 (1977).

17. P. Hohenberg and W. Kohn, Inhomogeneous electron gas // Phys. Rev. B, 136, p. 864-871 (1964).

18. W. Kohn and L.J. Sham, Self-consistent equations including exchange and correlation effects // Phys. Rev. A, 140, p. 1133-1138 (1965).

19. D.M. Ceperley and B.J. Alder, Ground state of the electron gas by a stochastic method // Phys. Rev. Lett. 45, p. 566-569 (1980).

20. J.P. Perdew and A. Zunger, Self-interaction correction to density-functional approximations for many-electron systems // Phys. Rev. B, 23, p. 50485079 (1981).

21. J.M. Soler, E. Artacho, J.D. Gale, A. Garcia, J. Junquera, P. Ordejon and D. Sanchez-Portal, The SIESTA method for ab initio order-N materials simulation // J. Phys.: Condens. Matter. 14, p. $2745-2779$ (2002).

22. http://www.icmab.es/siesta-joomla

23. G.B. Bachelet, D.R. Hamann and M. Schlüter, Pseudopotentials that work: From $\mathrm{H}$ to $\mathrm{Pu} / /$ Phys. Rev. B, 26, p. 4199-4228 (1982).

24. C. Hartwigsen, S. Goedecker and J. Hutter, Relativistic separable dual-space Gaussian pseudopotentials from H to Rn // Phys. Rev. B, 58, p. 3641-3662 (1998).

25. D.J. Chadi and M.L. Cohen, Special points in the Brillouin zone // Phys. Rev. B, 8, p. 5747-5753 (1973).

26. H.J. Monkhorst and J.D. Pack, Special points for Brillouin-zone integrations // Phys. Rev. B, 13, p. 5188-5192 (1976).

27. D. Foix, H. Martinez, A. Pradel, M. Ribes and D. Gonbeau, XPS valence band spectra and theoretical calculations for investigations on thiogermanate and thiosilicate glasses // Chem. Phys. 323, p. 606-616 (2006).

28. M.L. Cohen, Electronic charge densities in semiconductors // Science, 179, p. 1189-1195 (1973). 